\title{
Evaluation of the energy-saving performance of heat-resistant paint
}

\author{
Takashi Oda $^{1,2,{ }^{*}}$, Kimihiro Yamanaka ${ }^{1}$, Mitsuyuki Kawakami ${ }^{3}$ \\ ${ }^{1}$ System Design of Tokyo Metropolitan University, Tokyo, Japan \\ ${ }^{2}$ Nissin Sangyo Co., Ltd., Tokyo, Japan \\ ${ }^{3}$ Human Sciences of Kanagawa University, Kanagawa, Japan
}

Email address:

oda-takashi1@ed.tmu.ac.jp (T. Oda), kiyamana@tmu.ac.jp (K. Yamanaka),kawakamim@kanagawa-u.ac.jp (M. Kawakami)

\section{To cite this article:}

Takashi Oda, Kimihiro Yamanaka, Mitsuyuki Kawakami. Evaluation of the Energy-Saving Performance of Heat-Resistant Paint. Science Journal of Energy Engineering. Vol. 2, No. 5, 2014, pp. 53-57. doi: 10.11648/j.sjee.20140205.11

\begin{abstract}
We developed an empirical quantitative evaluation of the energy-saving performance of heat-resistant paint in winter. Specifically, heat-resistant paint and conventional wall paint were applied to steel boxes placed in a room kept at constant temperature and humidity, after which heat sources were placed in the boxes and the energy-saving performance of each type of paint was evaluated from the change in temperature at the box walls and inside the boxes. The experimental results show that the heat-resistant paint reduced the amount of heat escaping through the walls, and it can thus be expected to reduce heat loss. Furthermore, in the case of the heat-resistant paint, the amount of heat passing through the walls was $16 \%$ less than that in the case of the conventional paint. The conduction heat flux for the box with ceramic insulating paint was less than that for other boxes. It is thus thought that the thermal resistance of the ceramic insulation paint is higher than that of the other paints. We estimated the thermal resistance of each paint and found that thermal resistance of ceramic insulating paint was 12.4 times that of conventional energy-saving paint and twice that of high-reflectance paint.
\end{abstract}

Keywords: Energy-Saving, Heat-Resistant Paint, Comfortable Work Area

\section{Introduction}

In recent years, the importance of various approaches to save energy in residential and work spaces has increased as a result of apprehension about global warming and the electric power supply after the Great East Japan Earthquake[1]. Measures related to cooling and heating loads have become particularly important, and there is a trend toward improving building insulation to reduce the effect of the outside environment and to allow cooling and heating equipment to be used in an efficient manner[2]. With regard to the performance of building insulation, in the past, insulation materials were often used to direct the transfer of heat toward the inside of a structure. Recently, there has been a growing expectation that the application of heat-resistant or heat-insulating paint, which can improve energy-saving performance, can serve as a new energy-saving technique that differs from the conventional technique of installing insulation materials.[3] However, the different mechanism and properties of this technique mean that conventional methods of evaluating insulation performance cannot be used to evaluate the energy-saving performance. As a consequence, each manufacturer is presently using its own proprietary standards to evaluate energy savings. For end users selecting a product, the lack of common indicators of energy-saving performance obstructs the promotion of effective energy saving, and therefore, a common method of quantitative evaluation is desirable. Furthermore, although there have been numerous reports on the energy-saving performance of heat-resistant paint in summer, few such evaluations are available for winter.

Among energy-saving paints, many are so-called high-reflectance paints, which when applied to the roof and outer walls of a building can help regulate heat on the outside of the building by reflecting sunlight with high efficiency [4]. The standards JIS K 5602 "Determination of reflectance of solar radiation by paint film" and JIS K 5675 "High solar reflectance paint for roof" detail a quantitative method of evaluating high-reflectance paints $[5,6]$. The method described in these standards determines solar reflectance from the measurements of spectral reflectivity using the spectral radiation distribution of standard sunlight. In contrast to the situation for outdoor paints, there are energy-saving paints that are used on interior surfaces not exposed to solar radiation as a means of increasing cooling and heating efficiency, but there 
has been no agreement on a common standard with which to evaluate the energy-saving effect of an interior paint [7]. It is confirmed that the interior paint has insulation characteristics in simulation by a characteristic of penetration depth of far-infrared radiation [8].

Against this background, the present study directly measures the conduction heat fluxes of ceramic insulating paints with the greatest market share among energy-saving paints for indoor surfaces and two varieties of conventional energy-saving paint. A study of the physiological influence on human body by the ceramic insulation paint interior decoration painting is conducted [9]. From the results, a more unified verification of effectiveness is proposed. The verification estimates the heat-transfer inhibition effect of energy-saving paints intended for indoor surfaces, with the ultimate aim of quantitatively evaluating the effectiveness of paints. Additionally, the effects of high-reflectance paints are compared.

\section{Experimental Method}

Iron boxes (sides: $500 \mathrm{~mm}$; thickness: $1.6 \mathrm{~mm}$ ) were used in the present study. Iron was chosen as the base material because it is a thermally stable raw material with low thermal resistance and the effects of individual differences are thus minimized. A ceramic insulating paint, conventional energy-saving paint, and high-reflectance paint were each applied to the inside of a separate box to create three different samples. The specifications of the three paints are given in table 1 .

Table 1. Specifications of the three paints

\begin{tabular}{llll}
\hline & Conventional energy-saving paint & Ceramic insulative paint & High-reflectance paint \\
\hline Diluent & Water & Water & Water \\
Coating method & Spray & Spray & Spray \\
Number of times recoating & 2 & 2 & 2 \\
Standard application amount & $0.2 \sim 0.4 \mathrm{~kg} / \mathrm{m}^{2} / \mathrm{times}$ & $0.2 \sim 0.23 \mathrm{~kg} / \mathrm{m}^{2} / \mathrm{times}$ & $0.22 \mathrm{~kg} / \mathrm{m}^{2} / \mathrm{times}$ \\
Specific gravity & 1.24 & 0.78 & 1.24 \\
\hline
\end{tabular}

In the experiment, the conduction heat flux from the inside to the outside of a box and the structural temperature on the inside and outside of a point on the box ceiling were measured while heat was generated inside the iron box by a small fan heater (FH-120, FUKADAC); the setup is shown in figure 1 . The heat flux was measured by a heat flux sensor (MF-180, Eko Instruments), and the temperature inside and outside the box was measured by a thermocouple. The experiment was performed in a room with constant temperature and humidity. The ambient temperature set at $22{ }^{\circ} \mathrm{C}$. Measurements were taken starting $30 \mathrm{~min}$ after the start of operation of the heater and the thermal behavior was studied at 10-min intervals from then. Additionally, it was verified before the start of each trial that the box and its interior were at the same temperature as the soundings $\left(22{ }^{\circ} \mathrm{C}\right)$.

Figure 1 shows the installation point of the heat flux sensor for the measurement of the conduction heat flux (inside the box). The heat flux sensor was affixed with double-sided tape to the ceiling of the box, which was not directly exposed to the warm air from the small heater. The conduction heat flux to the outside was measured by a logger (3635-04, Hioki E. E. Corporation) at a sampling rate of 1 sample per minute while the heater was operating inside the box.

Figure 3 shows an iron box specimen and the installation points of the thermojunction temperature sensors (inside the box). The thermojunction temperature sensors were affixed to the inner and outer surfaces of the box ceiling with aluminum tape. The changes in the inside and outside surface temperatures of the box structure were measured by a logger (309, Centre) at a sampling rate of 1 sample per minute while the heater was operating inside the box.
Temperature-controlled room

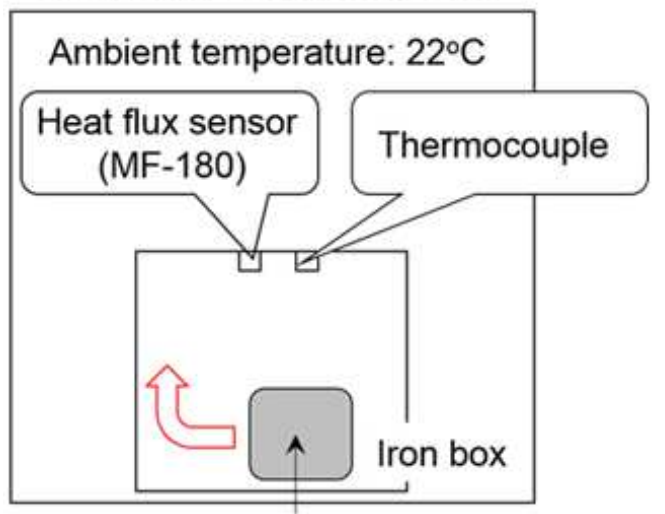

Small fan heater ( $\mathrm{FH}-1290)$

Figure 1. Experimental setup

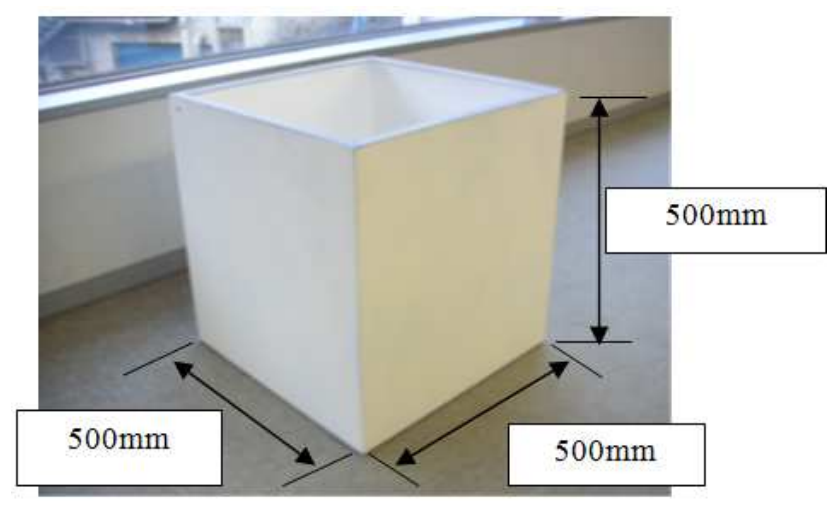

Figure 2. Iron box 


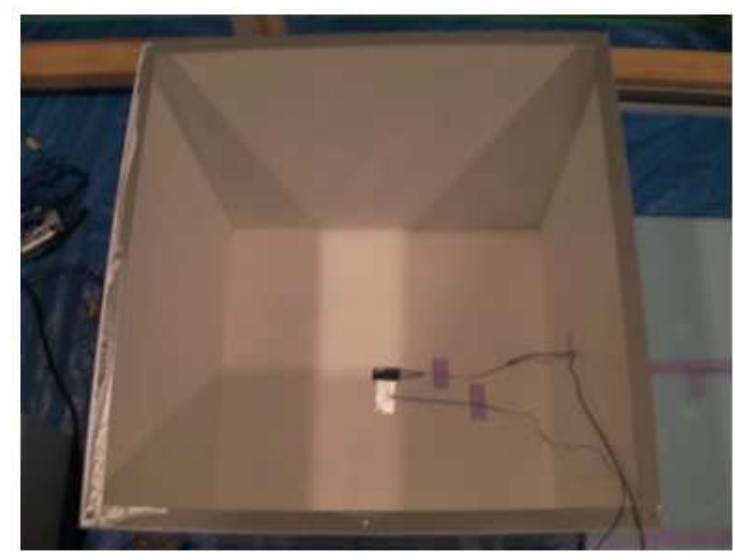

Figure 3. Installation points of the thermojunction temperature sensors (inside the box)

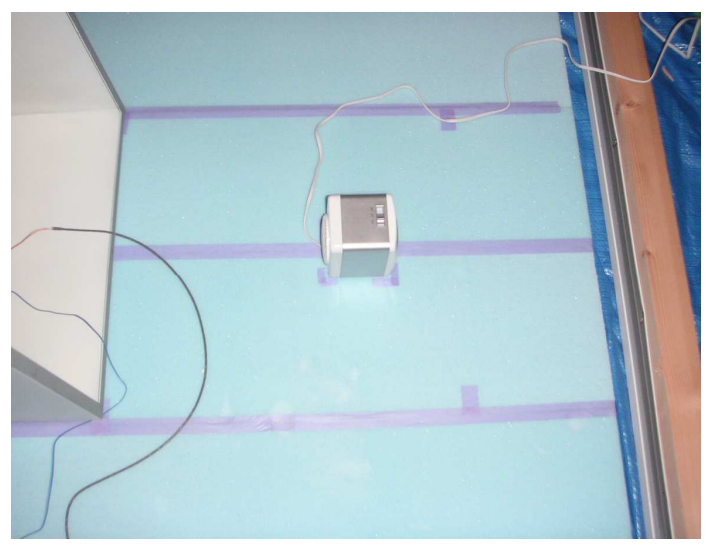

Figure 4. The setting situation

\section{Results for Conduction Heat Flux}

Figure 5 shows the conduction heat flux over time. The horizontal axis in the figure shows the elapsed time and the vertical axis shows the conduction heat flux from inside to outside the box. The three curves indicate the conduction heat fluxes of the three boxes. According to the figure, the trend in conduction heat flux appears to differ by paint between the first half and second half of the measurements. The measurement time was split into three periods (1-10 min, 11-20 $\mathrm{min}$, and 21-30 $\mathrm{min}$ ) to characterize these differences. Figure 6 shows the results of consolidating the conduction heat flux for each paint in each time period. The horizontal axis shows the time period, and the vertical axis shows the average conduction heat flux from inside to outside the box for each paint in each time period. The three bars show the results for each box. As can be seen in the figure, the conduction heat flux of the box to which ceramic insulating paint was applied tended to be smaller than that of other boxes in each time period. Table 2 gives the results of taking the values of the figure as characteristic values and conducting a two-way analysis of variance (ANOVA) with time period (A) and variety of paint (B) as factors. As seen in the table, both the measurement time period and the variety of paint were significant ( $\mathrm{p}<0.05$ and $\mathrm{p}<0.01$, respectively), but a pairwise comparison employing the Holm method showed that there were no significant differences in conduction heat flux among either the measurement time periods or the varieties of paint.

Table 2. ANOVA table for Conduction heat flux

\begin{tabular}{lllll}
\hline Source of variation & SS & df & MS & F-value \\
\hline Time period & 2066.88 & 2.00 & 1033.44 & $4.23 *$ \\
Variety of paint & 8267.54 & 2.00 & 4133.77 & $16.92 * *$ \\
Interaction & 2545.68 & 4.00 & 636.42 & 2.61 \\
Within & 19784.25 & 81.00 & 244.25 & \\
Total & 32664.35 & 89.00 & & \\
\hline
\end{tabular}

$(* *: \mathrm{p}<0.01, *: \mathrm{p}<0.05)$
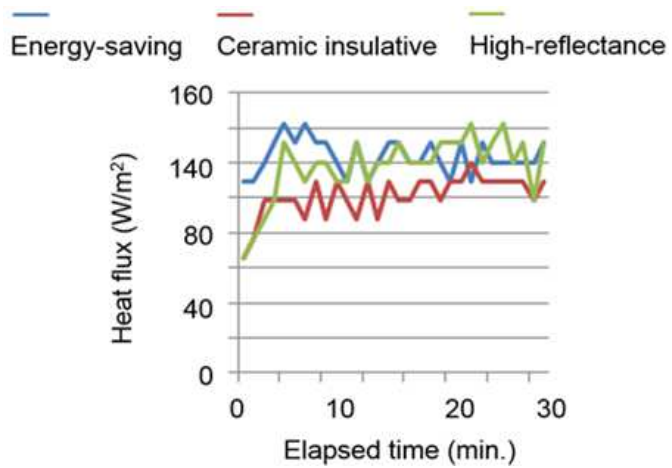

Figure 5. Results of measurement the Conduction heat flux

Energy-saving $\overline{\text { Ceramic insulative }}$ High-reflectance

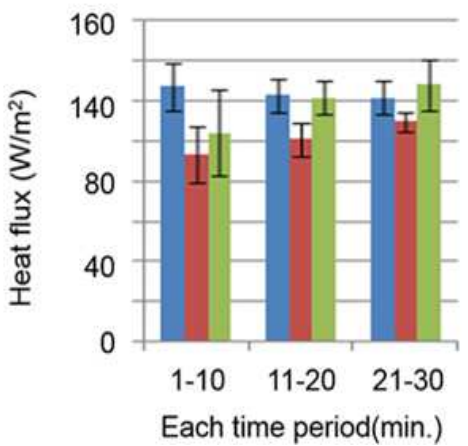

Figure 6. Results of the conduction heat in each time period

\section{Results for Structural Temperatures of the Inner and Outer Walls}

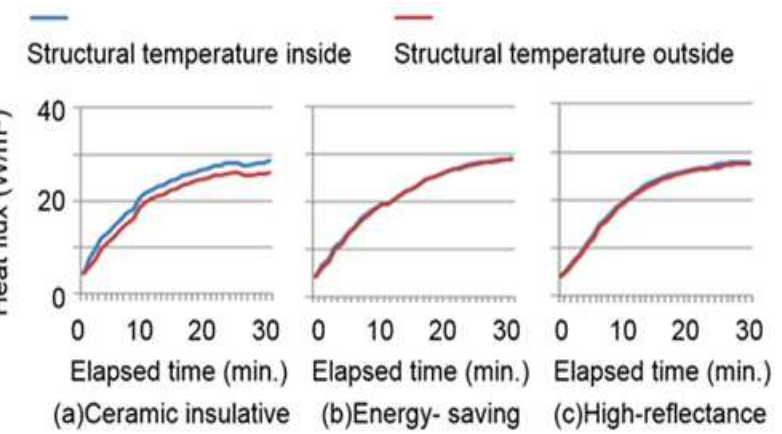

Figure 7. Changes in inner wall and outer wall temperatures 
Figure 7 shows the changes in temperatures of the inner and outer walls of the boxes. The horizontal axes in the figures show the elapsed time and the vertical axes show the wall surface temperature. The two curves in each figure show the temperature changes for the inner and outer walls.

The figure reveals that the temperature of the inner wall tended to be higher, from immediately after the heater was turned on, in the box to which ceramic insulating paint was applied, and there was a temperature gradient between the inside and outside of that box. In contrast, the temperatures of the inner and outer walls of the other boxes changed almost identically.

Figure 8 shows the inner-outer-wall temperature difference for each box in each time period. The horizontal axis in the figure shows the paint and the vertical axis shows the inner-outer-wall temperature difference. The three curves show results for different measurement time periods. Table 3 gives the results of taking the values of the figure as characteristic values and conducting a two-way ANOVA with time period (A) and variety of paint (B) as factors. As noted in the table, both the measurement time period and the variety of paint were significant $(\mathrm{p}<0.05$ and $\mathrm{p}<0.01$, respectively). Furthermore, pairwise comparison employing the Holm method showed that the inner-outer-wall temperature difference was significantly larger for the ceramic paint.

These results indicate that heat loss from the box with ceramic insulating paint was the lowest, which is consistent with the variety of paint being an influencing factor in the results of the conduction heat flux, as shown in table 2.

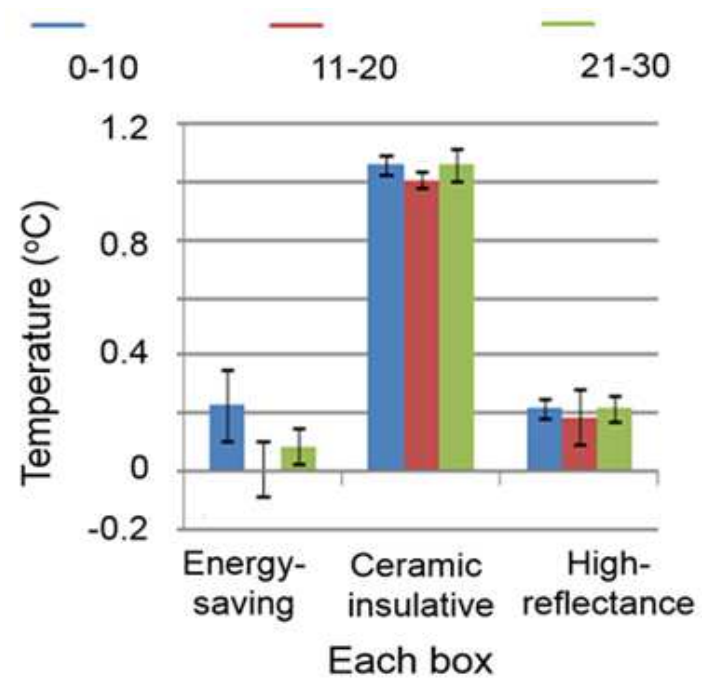

Figure 8. The inner-outer wall temperature difference

Table 3. ANOVA table for inner-outer wall

\begin{tabular}{lllll}
\hline Source of variation & SS & df & MS & F-value \\
\hline Time period & 2066.88 & 2.00 & 1033.44 & $4.23^{*}$ \\
Variety of paint & 8267.54 & 2.00 & 4133.77 & $16.92^{* *}$ \\
Interaction & 2545.68 & 4.00 & 636.42 & 2.61 \\
within & 19784.25 & 81.00 & 244.25 & \\
total & 32664.35 & 89.00 & & \\
\hline
\end{tabular}

\section{Estimate of the Thermal Resistance}

The conduction heat flux for the box with ceramic insulating paint was less than that for other boxes. It is thus thought that the thermal resistance level of the ceramic insulating paint is higher than that of other paint. We therefore estimate the thermal resistance of each paint in the following.

The thermal resistance $\mathrm{R}$ is expressed as [10]

$$
R=\Delta T / Q
$$

where $\Delta \mathrm{T}$ is the difference in temperature inside and outside the box and $\mathrm{Q}$ is the conduction heat flux.

We used expression (1) to estimate the thermal resistance of each paint. The data for the period 21-30 minutes after the beginning of heating are the amounts of heat flux and are relatively stable; we thus selected this time period for the calculation.

In general, the difference in structural temperature inside and outside the box becomes large as the insulation performance improves.

We estimated the thermal resistance of each box from the experimental data. The thermal resistance of the coating of each box was estimated by excluding the thermal resistance of the iron moiety. The thermal resistance levels were estimated as $0.0008 \mathrm{~m} 2 \cdot \mathrm{K} / \mathrm{W}$ for conventional energy-saving paint, $0.0100 \mathrm{~m} 2 \cdot \mathrm{K} / \mathrm{W}$ for ceramic insulating paint, and 0.0016 $\mathrm{m} 2 \cdot \mathrm{K} / \mathrm{W}$ for high-reflectance paint. The thermal resistance level of the ceramic insulating paint was 12.4 times that of the conventional energy-saving paint, and twice that of the high-reflectance paint. An explanation for the ceramic insulating paint having the highest thermal resistance requires an evaluation of the physical properties of the coating and remains a problem for future research.

\section{Conclusion}

The present study of the heat transfer inhibition effect of energy-saving paints experimentally verified the effects of three varieties of paint-an ordinary paint, a ceramic insulating paint, and a high-reflectance paint—applied to iron boxes. The results obtained are summarized as follows.

1) The variety of paint was shown to be an influencing factor of the conduction heat flux, which is an index that represents the loss of heat to the outside of the box.

2) The inside-outside temperature gradient of the box was steeper for the box with ceramic paint than for the other boxes, indicating that heat loss was smallest for the box with ceramic paint.

3) We estimated that the thermal resistance of the ceramic paint was the highest among the paints.

\section{References}

[1] Agency for Natural Resources and Energy "Summary of the law concerning the rational use of energy" http://www.enecho.meti.go.jp/category/saving_and_new/savin g/summary/pdf/2014_gaiyo.pdf (24/8/2014 access). 
[2] Ministry of Land, Infrastructure, Transport and Tourism "Energy-saving standard of a house and the building"http://www.mlit.go.jp/common/000996591.pdf (24/8/2014 access).

[3] SK KAKEN Co.,LTD "About the latest trend of the high reflectance paint"http://www.kenzai.or.jp/kouryu/image/42-04.pdf (24/9/2014 access).

[4] Japan Paint Manufacturers Association, "Guideline of manufacture and environment 2011", http://www. toryo.or.jp/ jp/anzen/reflect/reflect-info2.pdf, (30/6/2014 access).

[5] Japanese Industrial Standards, "Determination of reflectance of solar radiation by paint film”, JIS K5602, 2008.

[6] Japanese Industrial Standards, "High solar reflectance paint for roof" detail a quantitative method of evaluating high-reflectance paints”, JIS K 5675, 2011.

[7] Ministry of Land, Infrastructure, Transport and Tourism, "Energy-saving standard of architectural structure", ttp://www.mlit.go.jp/common/000996591.pdf, ， (01/7/2014 access).

[8] Takashi Oda, Masato Tazawa, Takeshi Kunishima, Kimihiro Yamanaka "Effect of penetration depth of far-infrared radiation into architectural walls" Grand renewable energy 2014

[9] Kazumi Tagami, Takashi Oda, Tastujiro Ishiko, Yuan Shenghua," Effects of inner wall thermal emissivity on human body temperature, and their metabolic heat production" Japanese Journal of Biometeorology 50 (3): S64, 2013

[10] Katsunori Hanamura"Practice-Heat transfer engineering", pp.11, 2007 